\title{
Effects of water activity on the performance of potassium sorbate and natamycin as preservatives against cheese spoilage moulds
}

\author{
P. Marín 1 , C . Ginés ${ }^{2}$, P. Kochaki², M. Jurado ${ }^{2 \dagger}$ \\ ${ }^{1}$ Plant Response Biotech S.L., Centro de Biotecnología y Genómica de Plantas, Campus de Montegancedo, 28223 Pozuelo de \\ Alarcón, Spain \\ ${ }^{2}$ Department of Chemistry and Food Technology, Escuela Técnica Superior de Ingeniería Agronómica, Alimentaria y de Biosistemas, \\ Universidad Politécnica de Madrid, Ciudad Universitaria s/n, 28040 Madrid, Spain
}

Abstract

This work investigated the effects of the food preservatives potassium sorbate and natamycin, combined with different levels of ionic (sodium chloride) and non-ioinic (glycerol) water activity (a $a_{w}$ ), on growth of fungi involved in cheese spoilage. In general, the combined effect of water stress and presence of preservatives enhanced fungal inhibition. However, some doses of potassium sorbate $(0.02 \%)$ and natamycin $(1,5$ and $10 \mathrm{ppm})$ were able to stimulate growth of Aspergillus varians, Mucor racemosus, Penicillium chrysogenum and $\mathrm{P}$. roqueforti at $a_{w}$ values in the range of 0.93-0.97. P. solitum was the only species whose growth was consistently reduced by any doses of preservative. The results also showed that sodium chloride and glycerol differentially affected the efficacy of preservatives. This study indicates that $a_{w}$ of cheese is a critical Keywords parameter to be considered in the formulation of preservative coatings used against fungal spoilage.

antifungals $\bullet$ cheese $\bullet$ food spoilage $\bullet$ preservation $\bullet$ water activity

\section{Introduction}

Mould spoilage is one of the major problems causing deterioration of cheese. Development of fungal growth can occur virtually at any point of the ripening and storage stages and may cause undesirable effects such as offodours and flavours, anomalous textures, discolourations and accumulation of mycotoxins (Sengun et al., 2008). Such defects are economically important since they are responsible for consumer rejection.

Different strategies can be implemented to improve the preservation of cheese and prevent the growth of moulds. A good cleaning and sanitising programme of the ripening rooms might help to reduce dispersible fungal spores present in the air and on the shelves (Ropars et al., 2012), although strict hygiene practices are difficult to maintain on a regular basis. In addition, cleaning and disinfecting might not be sufficient, considering that mould spores can also be transferred directly from milk to cheese (Lavoie et al., 2012; Panelli et al., 2014). Brushing of cheese wheels might help to remove mycelia from the rind but is a time-consuming activity, especially in the case of cheeses that are ripened for very long periods. Other practices, such as exclusion of air from the cheese through vacuum packaging, can also help to minimise mould growth. However, this procedure is normally done when cheeses have completed their ripening process, since vacuum packaging can slow down chemical and microbiological transformations necessary for the generation of volatile compounds (Andiç et al., 2011). In addition, vacuum packaging is not suitable for soft cheeses because it may induce undesirable changes in their textural properties (Pantaleão et al., 2007). The addition of food preservatives with fungistatic activity, though controversial, is still one of the best approaches for improving the keeping quality of cheese. Nevertheless, additives need to be used responsibly to ensure they comply with food regulations, especially those regarding the maximum usable dose. In most countries, the only preservatives authorised in cheese are natamycin and weak organic acids (sorbic or propionic acid) and their salts (sorbates and propionates) (Stark and Tan, 2003).

Besides preservatives, environmental factors might have a major impact on fungal growth. These factors include, among others, temperature, $\mathrm{pH}$ and availability of nutrients. Recently, it has been reported that water activity $\left(a_{w}\right)$ is a key factor that modulates the growth of fungi associated with cheese (Marin et al., 2014). The status of water in cheese is extremely variable and depends not only on the variety of cheese considered but also on the stage of maturation (Gaucel et al., 2012; Pajonk et al., 2003; Saurel et al., 2004). During cheese ripening, a number of complex processes that involve chemical and biochemical

${ }^{\dagger}$ Corresponding author: Miguel Jurado

E-mail:miguel.jurado@upm.es 
reactions, mainly proteolysis and lipolysis, are responsible for the accumulation of low-molecular-weight compounds (Duggan et al., 2008). These events, together with water loss and diffusion of the sodium chloride $(\mathrm{NaCl})$ added during manufacture, determine the occurrence of wide fluctuations in the concentration of ionic and non-ioinic osmolytes and, subsequently, on the levels of ionic and non-ionic $a_{w}$ (Duggan et al., 2008) This variability influences the distribution of mycobiota involved in cheese spoilage because fungal species are differentially affected by ionic and non-ionic compounds (Marín et al., 2014).

The study of the interaction between fungistatic preservatives and environmental factors might be useful for predicting their efficacy, and thus there have been a number of previous studies on this subject (Guynot et al., 2005; Huang et al., 2009; Marín et al., 2002; Suhr and Nielsen, 2004). However, to our knowledge, none of them have focussed on fungi associated with cheese. Therefore, the objective of the present study was to evaluate the effect of different regimes of ionic and non-ionic $a_{w}$ on the performance of potassium sorbate (PS) and natamycin as preservatives against mould species involved in cheese spoilage.

\section{Materials and methods}

\section{Preparation of media}

Sabouraud dextrose agar (Oxoid, Madrid, Spain) was used in this study. The value of $a_{w}$ was modified with the ionic solute $\mathrm{NaCl}$ or the non-ionic solute glycerol to $\mathrm{a}_{\mathrm{w}}$ values of 0.99 , $0.97,0.95$ and 0.93 . These solutes were not added to the control medium $\left(a_{w}=0.996\right)$. The $a_{w}$ of the media was checked with a hygrometer (AquaLab 3TE; Decagon Devices Inc., Pullman, Washington, USA).

PS (Sigma, Madrid, Spain) and natamycin of $50 \%$ purity (Danisco, Madrid, Spain) were aseptically added to the aforementioned media after autoclaving to give final concentrations of $0.02 \%, 0.1 \%$ and $0.2 \%$ (PS) or 1,5 and $10 \mathrm{ppm}$ (pure natamycin). No preservatives were added to the control media. The $\mathrm{pH}$ values of the media were adjusted to 5.4 with sterile $0.1 \mathrm{~mol} / \mathrm{L} \mathrm{HCl}$ after autoclaving, since the effectiveness of PS is known to be dependent on the $\mathrm{pH}$ (Plumridge et al., 2004).

\section{Fungal isolates}

Five fungal strains previously isolated from cheese (Marín et al., 2014) and comprising Aspergillus varians Mah1, Mucor racemosus Bet1, Penicillium solitum Mon2, Penicillium roqueforti Man1-3 and Penicillium chrysogenum Qpe1 were used for this study. Cultures were maintained on Sabouraud dextrose agar (Oxoid) at $4^{\circ} \mathrm{C}$ and stored as spore suspensions in $15 \%$ glycerol at $-20^{\circ} \mathrm{C}$.

\section{Inoculation, incubation and growth assessment}

A 5-mm-diameter agar disc from the margin of a 7-d-old growing colony of each isolate grown at $20^{\circ} \mathrm{C}$ was used to centrally inoculate each replicate and treatment. The plates were incubated at $20^{\circ} \mathrm{C}$ for $10 \mathrm{~d}$, and the experiment consisted of a fully replicated set of treatments with three replicates per treatment. Assessment of growth was made daily during the $10-d$ incubation period or until the colony reached the edge of the plate. Two diameters of the growing colonies were measured at right angles, and the radii of the colonies were plotted against time. Linear regressions were used to obtain growth rates from the slope of the line.

\section{Statistical analysis of results}

A two way analysis of variance (ANOVA) for each type of solute potential $\left(a_{w}\right.$ level $\times$ preservative dose) was performed separately for each species and fungistatic agent. A one-way ANOVA was performed when interaction of both factors ( $a_{w}$ $\times$ preservative dose) was significant. Subsequent post hoc analyses (Tukey's honest significance difference [HSD] tests of multiple comparisons) were carried out at a $95 \%$ confidence level. All sets of results were evaluated using Statgraphics Centurion XVII (Statistical Graphics Corp., Herndon, VA, USA) and SPSS 17.0.0 (Release 2008; SPSS Inc., Chicago, IL, USA).

\section{Results}

In general, and according to the two-way ANOVA performed separately for each fungal strain and preservative, the two factors (" $\mathrm{a}_{\mathrm{w}}$ level" and "preservative dose") - as well as their interaction - had statistically significant effects on fungal growth of all the species tested (data not shown). Subsequently, a one-way ANOVA and their corresponding Tukey's HSD tests were carried out to determine which values of growth rate were different at a statistically significant level (Tables 1-5).

The contour maps of the relative growth rates in response to different $\mathrm{a}_{\mathrm{w}}$ levels (0.996-0.93), $\mathrm{a}_{\mathrm{w}}$ types $(\mathrm{NaCl}$ or glycerol) and different doses of PS $(0 \%-0.2 \%)$ or natamycin $(0-$ $10 \mathrm{ppm}$ ) are shown in Figures 1-5. The standard deviations of the triplicates were typically $<10 \%$ of the mean. When no preservatives were added, the decrease of $a_{w}$ caused a different effect on fungal growth depending on the species tested. $M$. racemosus was the fastest-growing species but also the most affected by water stress, its growth being severely reduced at 0.95 and almost or completely inhibited at 0.93. Conversely, the reduction in $\mathrm{a}_{w}$ had a lower impact on growth of $P$. chrysogenum and no impact at all on growth of $P$. solitum.

The solute used to lower $\mathrm{a}_{\mathrm{w}}$ also influenced the growth rates at a statistically significant level, with $\mathrm{NaCl}$ being more inhibitory 
Table 1. Results of Tukey's HSD tests performed for $A$. varians growth rate $(\mathrm{mm} / \mathrm{d})$ and carried out separately for each factor (value of $\mathrm{a}_{\mathrm{w}}$ and dose of fungistatic) ${ }^{*}$

\begin{tabular}{|c|c|c|c|c|c|c|c|c|c|}
\hline \multicolumn{10}{|c|}{ A. varians Tukey's HSD tests } \\
\hline \multicolumn{10}{|c|}{ Potassium sorbate } \\
\hline & \multicolumn{5}{|c|}{ Value of $\mathrm{a}_{\mathrm{w}}(0 \% / 0.02 \% / 0.1 \% / 0.2 \%)$} & \multicolumn{4}{|c|}{ Dose of fungistatic $(0.996 / 0.99 / 0.97 / 0.95 / 0.93)$} \\
\hline & 0.996 & 0.99 & 0.97 & 0.95 & 0.93 & $0 \%$ & $0.02 \%$ & $0.1 \%$ & $0.2 \%$ \\
\hline $\mathrm{NaCl}$ & aaab & abcc & bac- & aaa- & aa-a & aabbc & acbde & abcb- & aa-a \\
\hline Glycerol & aaab & aabb & abc- & aabc & abc- & babcc & bacde & aabcc & aa-a- \\
\hline \multicolumn{10}{|c|}{ Natamycin } \\
\hline & \multicolumn{5}{|c|}{ Value of $a_{\mathrm{w}}(0 \mathrm{ppm} / 1 \mathrm{ppm} / 5 \mathrm{ppm} / 10 \mathrm{ppm})$} & \multicolumn{4}{|c|}{ Dose of fungistatic $(0.996 / 0.99 / 0.97 / 0.95 / 0.93)$} \\
\hline & 0.996 & 0.99 & 0.97 & 0.95 & 0.93 & $0 \%$ & $0.02 \%$ & $0.1 \%$ & $0.2 \%$ \\
\hline $\mathrm{NaCl}$ & $a b b b$ & $a b b b$ & baba & aaaa & cbaa & aabbc & abccc & abcbb & aaaaa \\
\hline Glycerol & abbb & aabb & aaaa & babc & $a b c c$ & babcc & babbc & aaaab & aaabc \\
\hline
\end{tabular}

${ }^{*}$ Means that are not significantly different from each other are represented with the same letter. Significant differences $(P<0.01)$ among means are indicated by different letters, where the ranking of letters corresponds to the ranking of means from highest to lowest $(a>b>c>d>e)$, and "-" indicates that no growth was detected.

$\mathrm{HSD}=$ honest significance difference.

Table 2. Results of Tukey's HSD tests performed for $M$. racemosus growth rate $(\mathrm{mm} / \mathrm{d})$ and carried out separately for each factor $(v a l u e$ of a and dose of fungistatic)*

\begin{tabular}{|c|c|c|c|c|c|c|c|c|c|}
\hline \multicolumn{10}{|c|}{ M. racemosus Tukey's HSD tests } \\
\hline \multicolumn{10}{|c|}{ Potassium sorbate } \\
\hline & \multicolumn{5}{|c|}{ Value of $a_{w}(0 \% / 0.02 \% / 0.1 \% / 0.2 \%)$} & \multicolumn{4}{|c|}{ Dose of fungistatic $(0.996 / 0.99 / 0.97 / 0.95 / 0.93)$} \\
\hline & 0.996 & 0.99 & 0.97 & 0.95 & 0.93 & $0 \%$ & $0.02 \%$ & $0.1 \%$ & $0.2 \%$ \\
\hline $\mathrm{NaCl}$ & aabb & abbc & aaab & $a b b-$ & - & aabc- & aabc- & aabc- & aaa- \\
\hline Glycerol & aabb & aabb & aabb & bacd & aab- & bbacd & cbade & aaabc & abbc- \\
\hline \multicolumn{10}{|c|}{ Natamycin } \\
\hline & \multicolumn{5}{|c|}{ Value of $a_{w}(0 \mathrm{ppm} / 1 \mathrm{ppm} / 5 \mathrm{ppm} / 10 \mathrm{ppm})$} & \multicolumn{4}{|c|}{ Dose of fungistatic $(0.996 / 0.99 / 0.97 / 0.95 / 0.93)$} \\
\hline & 0.996 & 0.99 & 0.97 & 0.95 & 0.93 & $0 \%$ & $0.02 \%$ & $0.1 \%$ & $0.2 \%$ \\
\hline $\mathrm{NaCl}$ & $a b b c$ & abcd & $a b c c$ & abba & - & aabc- & aabc- & $a b c c-$ & abdc- \\
\hline Glycerol & $a b b c$ & $a a b c$ & abcd & aaab & $a-$ & bbacd & baab- & baab- & aaab- \\
\hline
\end{tabular}

${ }^{\star}$ Means that are not significantly different from each other are represented with the same letter. Significant differences $(P<0.01)$ among means are indicated by different letters, where the ranking of letters corresponds to the ranking of means from highest to lowest $(a>b>c>d>e)$, and "-" indicates that no growth was detected.

$\mathrm{HSD}=$ honest significance difference.

Table 3. Results of Tukey's HSD tests performed for $P$. solitum growth rate $(\mathrm{mm} / \mathrm{d})$ and carried out separately for each factor (value of $\mathrm{a}_{\mathrm{w}}$ and dose of fungistatic)*

\begin{tabular}{|c|c|c|c|c|c|c|c|c|c|}
\hline \multicolumn{10}{|c|}{$P$. solitum Tukey's HSD tests } \\
\hline \multicolumn{10}{|c|}{ Potassium sorbate } \\
\hline & \multicolumn{5}{|c|}{ Value of $a_{w}(0 \% / 0.02 \% / 0.1 \% / 0.2 \%)$} & \multicolumn{4}{|c|}{ Dose of fungistatic $(0.996 / 0.99 / 0.97 / 0.95 / 0.93)$} \\
\hline & 0.996 & 0.99 & 0.97 & 0.95 & 0.93 & $0 \%$ & $0.02 \%$ & $0.1 \%$ & $0.2 \%$ \\
\hline $\mathrm{NaCl}$ & aаaа & aabb & $a b b b$ & abcd & $a a b-$ & aaaaa & baaaa & $c d a b c$ & bcac- \\
\hline Glycerol & aaaa & aabb & abcd & aabb & abcc & aaaaa & cbaab & baaab & aaaaa \\
\hline \multicolumn{10}{|c|}{ Natamycin } \\
\hline & \multicolumn{5}{|c|}{ Value of $a_{w}(0 \mathrm{ppm} / 1 \mathrm{ppm} / 5 \mathrm{ppm} / 10 \mathrm{ppm})$} & \multicolumn{4}{|c|}{ Dose of fungistatic $(0.996 / 0.99 / 0.97 / 0.95 / 0.93)$} \\
\hline & 0.996 & 0.99 & 0.97 & 0.95 & 0.93 & $0 \%$ & $0.02 \%$ & $0.1 \%$ & $0.2 \%$ \\
\hline $\mathrm{NaCl}$ & aaaa & abbc & abcc & $\mathrm{abcc}$ & aaaa & aaaaa & bbaab & aabaa & aaaaa \\
\hline Glycerol & aaaa & $a a b b$ & aabc & $a a b c$ & $a a b-$ & bbaab & ccaab & bbaab & aaaa- \\
\hline
\end{tabular}

${ }^{*}$ Means that are not significantly different from each other are represented with the same letter. Significant differences $(P<0.01)$ among means are indicated by different letters, where the ranking of letters corresponds to the ranking of means from highest to lowest $(a>b>c>d>e)$, and " - " indicates that no growth was detected.

$\mathrm{HSD}=$ honest significance difference. 
Table 4. Results of Tukey's HSD tests performed for P. chrysogenum growth rate $(\mathrm{mm} / \mathrm{d})$ and carried out separately for each factor (value of $\mathrm{a}_{\mathrm{w}}$ and dose of fungistatic)*

\begin{tabular}{|c|c|c|c|c|c|c|c|c|c|}
\hline \multicolumn{10}{|c|}{ P. chrysogenum Tukey's HSD tests } \\
\hline \multicolumn{10}{|c|}{ Potassium sorbate } \\
\hline & \multicolumn{5}{|c|}{ Value of $a_{w}(0 \% / 0.02 \% / 0.1 \% / 0.2 \%)$} & \multicolumn{4}{|c|}{ Dose of fungistatic $(0.996 / 0.99 / 0.97 / 0.95 / 0.93)$} \\
\hline & 0.996 & 0.99 & 0.97 & 0.95 & 0.93 & $0 \%$ & $0.02 \%$ & $0.1 \%$ & $0.2 \%$ \\
\hline $\mathrm{NaCl}$ & abcc & aabb & abbc & aabc & aa- & aaaab & baaab & bbab- & bbab- \\
\hline Glycerol & abcc & aabb & abcc & babc & aaab & baacc & bbacc & bbaab & baaab \\
\hline \multicolumn{10}{|c|}{ Natamycin } \\
\hline & \multicolumn{5}{|c|}{ Value of $a_{w}(0 \mathrm{ppm} / 1 \mathrm{ppm} / 5 \mathrm{ppm} / 10 \mathrm{ppm})$} & \multicolumn{4}{|c|}{ Dose of fungistatic $(0.996 / 0.99 / 0.97 / 0.95 / 0.93)$} \\
\hline & 0.996 & 0.99 & 0.97 & 0.95 & 0.93 & $0 \%$ & $0.02 \%$ & $0.1 \%$ & $0.2 \%$ \\
\hline $\mathrm{NaCl}$ & aabc & aabb & abcc & aabb & aacb & aaaab & babbb & aaaab & bbbaa \\
\hline Glycerol & aabc & $a a b c$ & $a b b c$ & babc & $a b c c$ & bbacc & aacbd & aaaab & bbbab \\
\hline
\end{tabular}

${ }^{*}$ Means that are not significantly different from each other are represented with the same letter. Significant differences $(P<0.01)$ among means are indicated by different letters, where the ranking of letters corresponds to the ranking of means from highest to lowest $(a>b>c>d>e)$, and " - " indicates that no growth was detected.

$\mathrm{HSD}=$ honest significance difference.

Table 5. Results of Tukey's HSD tests performed for $P$. roqueforti growth rate $(\mathrm{mm} / \mathrm{d})$ and carried out separately for each factor (value of $\mathrm{a}_{\mathrm{w}}$ and dose of fungistatic)*

\begin{tabular}{|c|c|c|c|c|c|c|c|c|c|}
\hline \multicolumn{10}{|c|}{ P. roqueforti Tukey's HSD tests } \\
\hline \multicolumn{10}{|c|}{ Potassium sorbate } \\
\hline & \multicolumn{5}{|c|}{ Value of $\mathrm{a}_{\mathrm{w}}(0 \% / 0.02 \% / 0.1 \% / 0.2 \%)$} & \multicolumn{4}{|c|}{ Dose of fungistatic $(0.996 / 0.99 / 0.97 / 0.95 / 0.93)$} \\
\hline & 0.996 & 0.99 & 0.97 & 0.95 & 0.93 & $0 \%$ & $0.02 \%$ & $0.1 \%$ & $0.2 \%$ \\
\hline $\mathrm{NaCl}$ & aabc & aabb & aaaa & aaa- & aa - & aabcd & bacde & aabc- & aaa- \\
\hline Glycerol & aabc & aaab & abcc & $\mathrm{Babb}$ & baaa & baacd & babac & babcd & baabc \\
\hline \multicolumn{10}{|c|}{ Natamycin } \\
\hline & \multicolumn{5}{|c|}{ Value of $\mathrm{a}_{\mathrm{w}}(0 \mathrm{ppm} / 1 \mathrm{ppm} / 5 \mathrm{ppm} / 10 \mathrm{ppm})$} & \multicolumn{4}{|c|}{ Dose of fungistatic $(0.996 / 0.99 / 0.97 / 0.95 / 0.93)$} \\
\hline & 0.996 & 0.99 & 0.97 & 0.95 & 0.93 & $0 \%$ & $0.02 \%$ & $0.1 \%$ & $0.2 \%$ \\
\hline $\mathrm{NaCl}$ & $a b c-$ & $a b-$ & $a b-$ & $a-$ & $a-$ & aabcd & $a c b-$ & $a-$ & 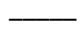 \\
\hline Glycerol & $a b c-$ & $a b c c$ & $a b c-$ & $a b c-$ & $a b-$ & baacd & aaabc & abbb- & $-a-$ \\
\hline
\end{tabular}

${ }^{*}$ Means that are not significantly different one each other are represented with the same letter. Significant differences $(P<0.01)$ among means are indicated by different letters, where the ranking of letters corresponds to the ranking of means from highest to lowest $(a>b>c>d>e)$, and "-" indicates that no growth was detected.

HSD $=$ honest significance difference.

than glycerol, especially in the case of $M$. racemosus and $P$. solitum. On the contrary, $P$. chrysogenum was the most halotolerant species.

The " $a_{w}$ level $\times$ preservative dose" interactions typically resulted in a higher inhibition of fungal growth. The growth rate decrease was, however, not always proportional to the reduction in $a_{w}$ level nor to the increase in concentration of fungistatic. As a result, the shape of the growth rate curves were different in the media supplemented with $\mathrm{NaCl}$ or glycerol in comparison to control media $\left(a_{w}=0.996\right)$ in the five species analysed.

Moreover, the use of certain doses of preservative at low $a_{w}$ levels (in the range of $0.93-0.97$ ) even resulted in a stimulation of fungal growth in $A$. varians $(0.02 \% \mathrm{PS}, 0.97$ $\mathrm{a}_{\mathrm{w}, \mathrm{N}} \mathrm{NaCl} ; 1$ and $10 \mathrm{ppm}$ natamycin, $0.97 \mathrm{a}_{\mathrm{w}, \mathrm{NaCl}} 1 \mathrm{ppm}$ natamycin, $0.95 \mathrm{a}_{\mathrm{w}}$, glycerol; 1, 5 and $10 \mathrm{ppm}$ natamycin, $\left.0.93 \mathrm{a}_{w}, \mathrm{NaCl}\right)$, M. racemosus (0.02\% PS, $0.95 \mathrm{a}_{w}$, glycerol),

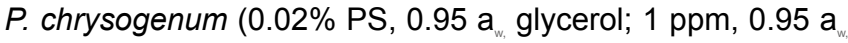
$\mathrm{NaCl})$ and $P$. roqueforti $\left(0.02 \% \mathrm{PS}, 0.95 \mathrm{a}_{w}\right.$, glycerol; $0.02 \%$, 0.1 and $0.2 \%$ PS, $0.93 \mathrm{a}_{\mathrm{w},}$ glycerol).

\section{Discussion}

Fungal spoilage of cheese occurs when moulds carried in milk or present in the chamber rooms are able to colonise the cheese rind, producing visible deterioration. Thus, spore germination and mycelial growth are subjected, on the one hand, to compositional intrinsic characteristics that depend on the cheese variety and, on the other hand, to the occurrence of extrinsic factors imposed from the outside. Study of the influence exerted by these factors is essential to 


\section{Aspergillus varians}

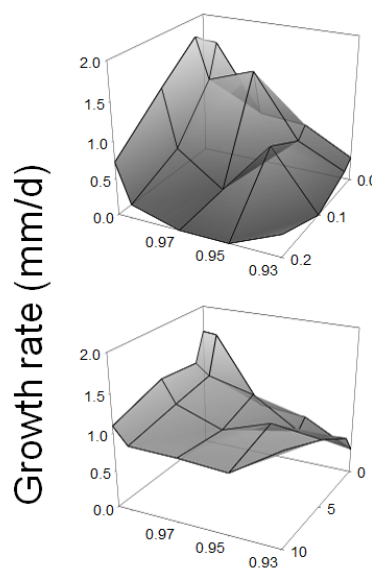

$\mathrm{a}_{\mathrm{w}}(\mathrm{NaCl})$

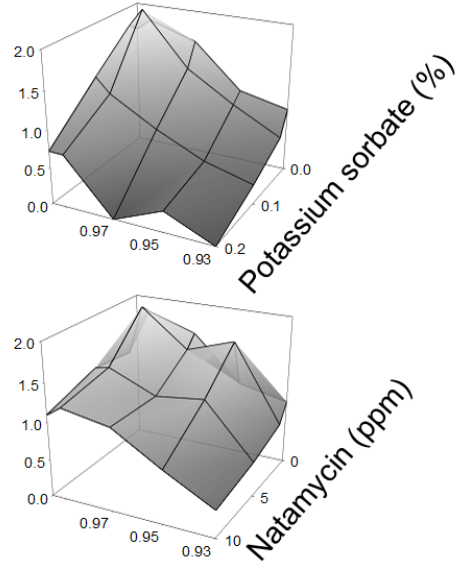

$a_{w}$ (glycerol)

Figure 1. Contour maps of the effect of $\mathrm{NaCl}$ or glycerol on the growth rates of Aspergillus varians incubated for $10 \mathrm{~d}$ at different doses of the fungistatic agents potassium sorbate or natamycin and different $a_{w}$ levels. The data shown are the mean of three replicates.

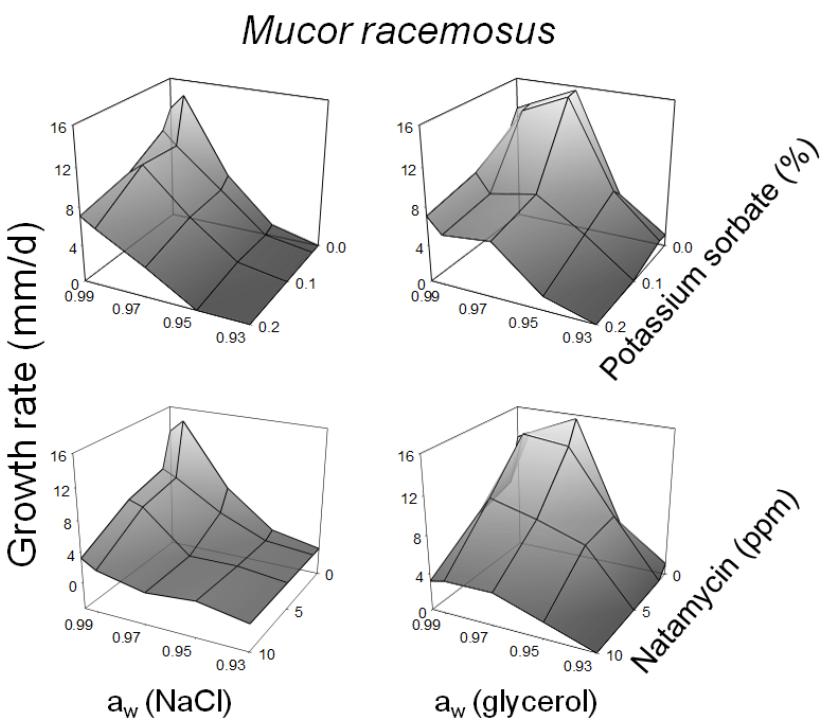

Figure 2. Contour maps of the effect of $\mathrm{NaCl}$ or glycerol on the growth rates of Mucor racemosus incubated for $10 \mathrm{~d}$ at different doses of the fungistatic agents potassium sorbate or natamycin and different $a_{w}$ levels. The data shown are the mean of three replicates.

predict the microbial stability of the product. According to the "Hurdle effect" concept (Leistner, 1992), many factors can be individually unable to completely inhibit microbial growth but the effectiveness can be enhanced when several factors occur simultaneously. In this study, we focussed on preservative concentration and $\mathrm{a}_{w}$ level because both factors might undergo quantitative fluctuations during cheese ripening and storage

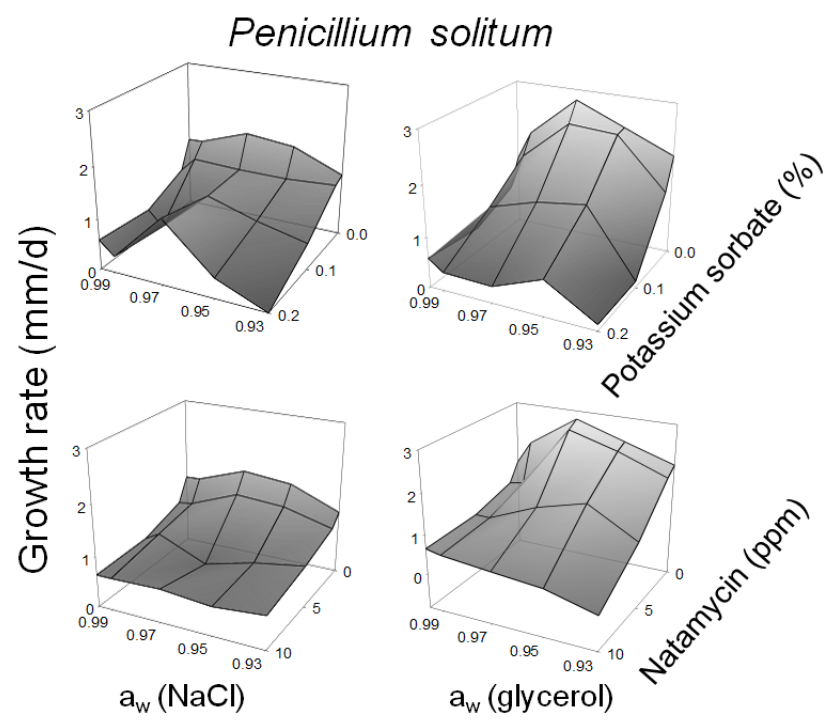

Figure 3. Contour maps of the effect of $\mathrm{NaCl}$ or glycerol on the growth rates of Penicillium solitum incubated for $10 \mathrm{~d}$ at different doses of the fungistatic agents potassium sorbate or natamycin and different $a_{w}$ levels. The data shown are the mean of three replicates.

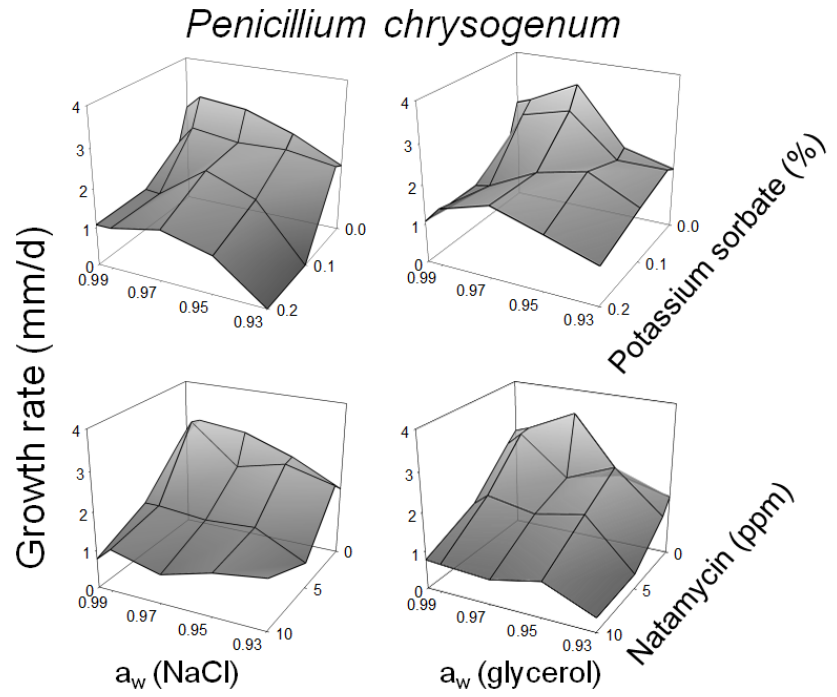

Figure 4. Contour maps of the effect of $\mathrm{NaCl}$ or glycerol on the growth rates of Penicillium chrysogenum incubated for $10 \mathrm{~d}$ at different doses of the fungistatic agents potassium sorbate or natamycin and different $a_{w}$ levels. The data shown are the mean of three replicates.

and thus might potentially influence the efficacy of these hurdles in preventing fungal spoilage. The concentrations at which preservatives are normally used in cheese are $0.02 \%-$ $0.15 \%$ in the case of sorbates (Azza and Ahmed, 2010) and 1-20 ppm in the case of natamycin (Kallinteri et al., 2013; Stark and Tan, 2003). The doses evaluated in this work might be therefore considered as ranging from standard to sub- 
optimal. The study of the effect of low doses of preservatives is of interest not only because the modern trend is to reduce their concentration in foodstuffs due to consumer demands, but also because concentration of fungistatics can decrease due to diffusion phenomena within the bulk of cheese (Kristo et al., 2008). It should be noted that PS can be either incorporated in the whole cheese mass or impregnated in the rind, whereas natamycin is exclusively permitted for surface treatment (European Food Safety Agency [EFSA], 2009). In addition, the active concentration of PS can be reduced due to degradation of the molecule by fungal and bacterial activity (Mann and Beucheat, 2008; Montaño et al., 2013), whereas natamycin is broken down by UV light (Pedersen, 1992).

The results obtained in this work showed that, in general, both PS and natamycin were more efficient in inhibiting fungal growth when $a_{w}$ was low. However, certain particular combinations of preservative doses and low $a_{w}$ values resulted in the reduced efficacy of the preservative, and some combinations even stimulated fungal growth. Particularly, the lowest tested dose of PS, combined with certain low $a_{w}$ values, was able to enhance fungal growth of $A$. varians, $M$. racemosus, $P$. chrysogenum and $P$. roqueforti. These results indicate that control of these moulds might be thus achievable through the addition of higher concentrations of the preservative. However, apart from the legal restrictions concerning the maximum permitted level of sorbates, the adverse effect of off-aromas and off-flavours that might result could make this approach impractical (Mann and Beucheat, 2008). Interestingly, a previous study that examined the interactions between PS and $\mathrm{a}_{w}$ on inhibition of fungi associated with bakery products also found a similar growth-stimulating effect of sub-optimal levels of PS at low $a_{w}$ values (Marín et al., 2002). It is well known that some fungi are able to tolerate low concentrations of PS because they can degrade the molecule through decarboxylation and use it as a source of carbon (Montaño et al., 2013). It can be hypothesised that low $a_{w}$ might selectively induce PS assimilation routes in some fungi, since there is increasing evidence that numerous metabolic pathways are differently expressed under different regimes of $a_{w}$ (Bai et al., 2015; Zhang et al., 2014; Zhang et al., 2015). This field of research deserves further exploration.

At some values of reduced ionic and non ionic $\mathrm{a}_{\mathrm{w}}$, the species $A$. varians, $M$. racemosus and $P$. chrysogenum showed evidence of non-monotonic dose-growth response to natamycin. This kind of response is characterised by a curve whose slope changes direction within the range of tested doses, in this case - with low and high levels, causing less inhibition (or more stimulation) of fungal growth than intermediate levels. Non-monotonic curves are usually explained as the result of superimposition of monotonic dose responses of the component biological reactions (Conolly and Lutz, 2004).

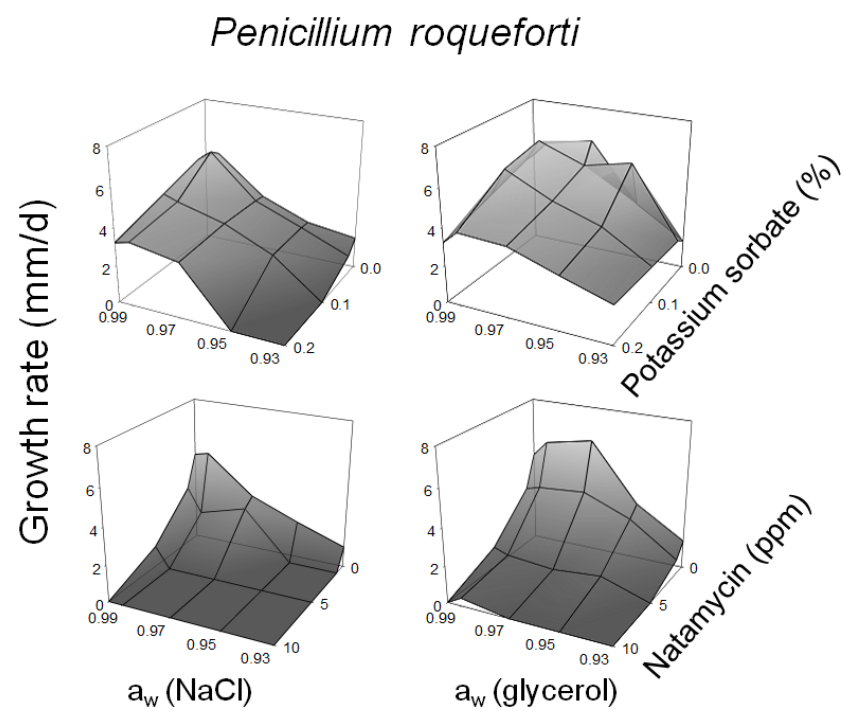

Figure 5. Contour maps of the effect of $\mathrm{NaCl}$ or glycerol on the growth rates of Penicillium roqueforti incubated for $10 \mathrm{~d}$ at different doses of the fungistatic agents potassium sorbate or natamycin and different $a_{w}$ levels. The data shown are the mean of three replicates.

Whatever the precise causes, our results suggest that in order to effectively inhibit these species, it could be necessary to ensure a stable concentration of natamycin. Strategies such as encapsulation of the preservatives to achieve a sustained release of the molecule (Gortzi et al., 2006) and the use of polymeric substances able to prevent its migration (Fajardo et al., 2010) could be useful for this purpose.

The data obtained in this work suggest that, in the presence of sub-optimal or standard doses of preservative, the natural decrease of $a_{w}$ that occurs during the ripening stage might trigger the growth of some fungal species if a certain threshold value is reached. It is possible that such a fact could be misinterpreted by cheese manufacturers as a decrease in the active concentration of the preservative, which could lead to unnecessary - and even counterproductive - re-application of preservative coatings. In our experience, re-application of preservative coatings is a very common practice in the cheese industry after an episode of re-emergence of mould contamination, especially in those varieties that are ripened for long periods of time. We think that both the formulation of preservative coatings and the timing of their application (and re-application) should be factors to carefully consider in any cheese industry.

Future research directed towards investigating the molecular mechanisms underlying resistance to food preservatives under water stress could also be beneficial. However, we must admit that this approach is overly complex, since the resistances detected varied according to the type of solute potential (ionic or non-ionic), as well as according to the species. Therefore, unfortunately, it seems very unlikely that guidelines in the 
management of preservatives can be applicable to a wide range of cheeses. This highlights the necessity of gaining knowledge about the taxonomy of the spoiling mycobiota associated with a particular cheese variety. Nonetheless, a better understanding of the environmental factors modulating the dynamics of fungal populations in cheese would be determinant to achieving a higher level of performance of preservatives. The integration of this information could be extremely useful to develop ad hoc preservative formulations against fungal spoilage of different varieties of cheese.

\section{References}

Andiç, S., Tunçtürk, Y. and Gençcelep, H. 2011. The effect of different packaging methods on the formation of biogenic amines and organic acids in Kashar cheese. Journal of Dairy Science 94: 1668-1678.

Azza, M.M. and Ahmed, H.F. 2010. Effect of potassium sorbate and/or probiotic bacteria on spoilage bacteria during cold storage of soft cheese. Global Veterinaria 4: 483-488.

Bai, Y., Lan, F., Yang, W., Zhang, F., Yang, K., Li, Z., Gao, P. and Wang, S. 2015. sRNA profiling in Aspergillus flavus reveals differentially expressed miRNA-like RNAs response to water activity and temperature. Fungal Genetics and Biology 81: 113-119.

Conolly, R.B. and Lutz, W.K. 2004. Nonmonotonic dose-response relationships: mechanistic basis, kinetic modeling, and implications for risk assessment. Toxicology Science 75: 151-157.

Duggan, E., Noronha, N., O'Riordan, E.D. and O'Sullivan, M. 2008. Effect of resistant starch on the water binding properties of imitation cheese. Journal of Food Engineering 84: 108-115.

EFSA (European Food Safety Agency). 2009. Scientific opinion on the use of natamycin (E 235). EFSA Journal 7: 1412.

Fajardo, P., Martins, J.T., Fuciños, C., Pastrana, L., Teixeira, J.A. and Vicente, A.A. 2010. Evaluation of a chitosan-based edible film as carrier of natamycin to improve the storability of Saloio cheese. Journal of Food Engineering 101: 349-356.

Gaucel, S., Guillemin, H. and Corrieu, G. 2012. A generalised model for cheese mass loss determination during ripening. Journal of Food Engineering 110: 109-116.

Gortzi, O., Lalas, S., Chiou, I. and Tsaknis, J. 2006. Reevaluation of antimicrobial and antioxidant activity of Thymus spp. extracts before and after encapsulation in liposomes. Journal of Food Protection 69: 2998-3005.

Guynot, M.E., Ramos, A.J., Sanchis, V. and Marín, S. 2005. Study of benzoate, propionate, and sorbate salts as mould spoilage inhibitors on intermediate moisture bakery products of low $\mathrm{pH}$ (4.5-5.5). International Journal of Food Microbiology 101: 161-168.

Huang, Y., Chapman, B., Wilson, M. and Hocking, A.D. 2009. Effect of agar concentration on the matric potential of glycerol agar media and the germination and growth of xerophilic and non-xerophilic fungi. International Journal of Food Microbiology 133: 179-185.
Kallinteri, L.D., Kostoula, O.K. and Savvaidis, I.N. 2013. Efficacy of nisin and/or natamycin to improve the shelf-life of Galotyri cheese. Food Microbiology 36: 176-181.

Kristo, E., Koutsoumanis, K.P. and Biliaderis, C.G. 2008. Thermal, mechanical and water vapor barrier properties of sodium caseinate films containing antimicrobials and their inhibitory action on Listeria monocytogenes. Food Hydrocolloid 22: 373-386.

Lavoie, K., Touchette, M., St-Gelais, D. and Labrie, S. 2012. Characterization of the fungal microflora in raw milk and specialty cheeses of the province of Quebec. Dairy Science and Technology 92: 455-468.

Leistner, L. 1992. Food preservation by combined methods. Food Research International 25: 151-158.

Mann, D.A. and Beucheat, L.R. 2008. Combinations of antimycotics to inhibit the growth of molds capable of producing 1,3-pentadiene. Food Microbiology 25: 144-153.

Marín, S., Guynot, M.E., Neira, P., Bernado, M., Sanchis, V. and Ramos, A.J. 2002. Risk assessment of the use of sub-optimal levels of weak-acid preservatives in the control of mould growth on bakery products. International Journal of Food Microbiology 79: 203-2011.

Marín, P., Palmero, D. and Jurado, M. 2014. Effect of solute and matric potential on growth rate of fungal species isolated from cheese. International Dairy Journal 36: 89-94.

Montaño, A., Sánchez, A.H., Casado, F.J., Beato, V.M. and de Castro, A. 2013. Degradation of ascorbic acid and potassium sorbate by different Lactobacillus species isolated from packed green olives. Journal of Food Microbiology 34: 7-11.

Pajonk, A.S., Saurel, R. and Andrieu, J. 2003. Experimental study and modeling of effective $\mathrm{NaCl}$ diffusion coefficients values during $\mathrm{Em}$ mental cheese brining. Journal of Food Engineering 60: 307-313.

Panelli, S., Brambati, E., Bonacina, C. and Feligini, M. 2014. Updating on the fungal composition in Sardinian sheep's milk by cultureindependent methods. Journal of Dairy Research 81: 233-237.

Pantaleão, I., Pintado, M.M.E. and Poças, M.F.F. 2007. Evaluation of two packaging systems for regional cheese. Food Chemistry 102: 481-487.

Plumridge, A., Stephan, J.A., Hesse, A., Watson, J., Lowe, K.C., Stratford, M. and Archer, D.B. 2004. The weak acid preservative sorbic acid inhibits conidial germination and mycelial growth of Aspergillus niger through intracellular acidification. Applied and Environmental Microbiology 70: 3506-3511.

Ropars, J., Cruaud, C., Lacoste, S. and Dupont, J. 2012. A taxonomic and ecologic overview of cheese fungi. International Journal of Food Microbiology 155: 199-210.

Saurel, R., Pajonk, A. and Andrieu, J. 2004. Modelling of French Emmental cheese water activity during salting and ripening periods. Journal of Food Engineering 63: 163-170.

Sengun, I., Yaman, D.B. and Gonul, S.A. 2008. Mycotoxins and mould contamination in cheese: a review. World Mycotoxin Journal 1: 291-298.

Stark, J. and Tan, H.S. 2003. Natamycin. In: "Food Preservatives" 
(eds. N.J. Russel and G.W. Gould), Kluwer Academic/Plenum Publishers, Dordrecht, Netherlands, pages 179-195.

Suhr, K.I. and Nielsen, P.V. 2004. Effect of weak acid preservatives on growth of bakery product spoilage fungi at different water activities and $\mathrm{pH}$ values. International Journal of Food Microbiology 95: $67-78$.

Pedersen, J.C. 1992. Natamycin as a fungicide in agar media. Applied and Environmental Microbiology 58: 1064-1066.
Zhang, F., Guo, Z., Zhong, H., Wang, S., Yang, W., Liu, Y. and Wang, S. (2014). RNA-Seq-based transcriptome analysis of aflatoxigenic Aspergillus flavus in response to water activity. Toxins 6: 31873207.

Zhang, F., Zhong, H., Han, X., Guo, Z., Yang, W., Liu, Y., Yang, K., Zhuang, Z. and Wang, S. 2015. Proteomic profile of Aspergillus flavus in response to water activity. Fungal Biology 119: 114-124. 